\title{
Climate Features of Mountainous Regions and Its Effect on Architecture Designation, Case Study of Residential Buildings of Bijar, Iran
}

\author{
Narges Zangeneh \\ Department of Architecture, Hidaj Branch, Islamic Azad University, Hidaj, Iran
}

Email address:

Narges1655@yahoo.com

To cite this article:

Narges Zangeneh. Climate Features of Mountainous Regions and Its Effect on Architecture Designation, Case Study of Residential Buildings of Bijar, Iran. International Journal of Science, Technology and Society. Special Issue: Research and Practice in Architecture and Urban Studies in Developing Countries. Vol. 3, No. 2-1, 2015, pp. 95-98. doi: 10.11648/j.ijsts.s.2015030201.28

\begin{abstract}
In order to determine the climate, we should have an accurate definition of climate and especially cold regions climate. The climate is a set of prevance conditions of climate and regions if mountainous areas have a cold atmosphere which should use fossil fuels energy for heating in the cold seasons to achieve relief. This leads to architecture designate apr Canvas using clean energies and materials with high heat coefficient which at all sustainable architecture designate should be considered. Bijar city clue to have a cold regions features plays key roles in architecture designate of its buildings and in recent years most of Iranian architects wants to use from this pattern but clute to unknowing the regions features, environment and native materials , they cannot reach it. The offered solution is that to designate in catch region, first we should analyze environment features and then with modern technologies and regard 19 discussion of national regulations of buildings and integration of these discussions, we can make building with the modern technologies against atmosphere conditions, in cold mountainous areas.
\end{abstract}

Keywords: Climate, Architecture, Cold Mountainous Regions

\section{Introduction}

Knowing of regions abilities in country architecture is basis of using past regional techniques regarding to the new technology according with stable architecture origins. The manner encounter by the nature in past architecture is indicator of humans on the factors such as regions incompatibility; lack of energy to achieve tranquility conditions architecture climate measures of country architecture has its maximum efficiency in its time and has overlap with the sustainable architectures bases. So, because the human being at past build a building such that not only were the most compatible each climate but also used from useful regions.

Factors like sunshine, the weather temperature, moisture, rainfall and wind to provide rest. In the buildings the considered region in above paper is mountainous region. Sam \& Chang (1997) carried out important investigations about using climate elements in architecture design \& building energy, they recommended using local climate conditions to improve climate designs \& building energy simulations [4]. Using monthly chart during 25 years period, Kafa analyzed climate elements of Nikozaki in Qebres to prepare general \& suitable data for optimum use of passive solar energies in urban planning and building design, then calculated and proposed pre-design strategies [9].

Toi et al 2007 investigated bioclimatic conditions in Arzerom, Turkey, in three areas including rural, urban and urban-Forest and concluded that urban-forest area is more compatible with utilized heat relaxation indexes [10]. Jonson 2006 investigated the effect of urban geometry on external heat relaxation in heat-dry climate and he concluded that in heat-dry areas urban design should be compacted in order to meet heat relaxation of the area. [11] In Iran, many studies have investigated the role of climate in architecture, building designs and urban designs. Using climate elements and climate graphs, most of them investigated concordant architecture with climate in various climates of Iran.

\section{The Case Study Region}

A set of predominant climate condition is called region. Usually in a big area in which the natural factors generate same climate predominant condition. the single region is 
made . Some parts on the region which has same climate condition is called micro- region.

The geographical factors like mountain, sea, lake, plain and so on lead to different parts of a region and micro- region. The regions factors based on their effect are classified in two groups. The main regions factors which include sunshine, weather temperature. Moisture, rainfall and wind and secondary regions agents that contain height from the sea level, distance from the sea and geographical tons.

The main climate factors

1) Sunshine: its origin is sun and is the most fundamental energy resource in the earth. The effect of sun in the architecture designation and buildings components the first effect is on the building orientation and its surface. West and east orientation of the building will have maximum absorption of light beams that if we want to absorb useful light. It is better interested in this tension (fig1).

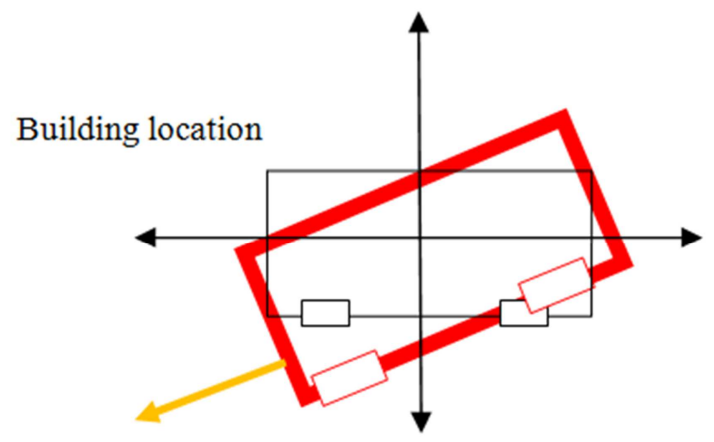

Best location

Fig. 1. The proposed positioning

It influences the texture quality of flamed ones.- The surface in dry and hot region should be translucent and dear and in the cold and mountainous areas should be dark rough.

It is adequate to choose the shined colors.

The light colours may reflect $90 \%$ of sun energy and dark colours only reflect $15 \%$ of sun energy. It effects on the number and space of the windows.

Assuming that the surfaces are same, the more windows, the more heat transfers because heating resistance of glass between the materials is very low.

It affects the structure and frame of the roof. As the roof during the day has the maximum absorption of the sun beams, at night it is cold rapidly by emitting the heating beams and so in the cold areas or winter. The transfer level of heat depends on the roof material and in the hot regions and hot weather; the main roof is the basic controller of inside air. In monolith roofs or combined ones that are transfer through conduction, we can say that (1) in these roofs dark colours has the surface temperature to $320 \mathrm{c}$ which is more than the maximum environment temperature and in the light roofs it reaches to $10 \mathrm{c}$ and of course heating capability and thickness influence from sun heating absorption. it mean that, the more heat capability and thickness the low roofs temperature difference in effect of different colours .(2). The location of insulation layer is useful. We should pay attention that adding insulation layer is useful. We should pay attention that adding insulation layer affects decreasing inside temperature.

It influence the apace height. Declining the roof height regardless economy causes to decrease inside volume and so lower cooling or heating costs also and speed up this process.

2) Moisture: the water that is in that is from of moisture in the weather is called moisture. The effect of the moisture on the building and architecture generally. The moisture is harmful for buildings materials and causes the decline the buildings life. We can categorize its effect as follow:

Humid walls make some illnesses resonance such as cold and rheumatiz. Humid walls have lower heating resistance and by declining the temperature of inside surface of the walls, the hydrolysis is intensified.

The moisture resolve salt minerals and exiting surface in it and then they are appeared as nitrate on the surface of wall each regardless to homeliness of face, cause to bad aroma in the apace. Humid damage buildings material like decrease resistance, corrosion in metal, changing woods dimension and so on.

3) Wind: difference between 2 regions causes difference in pressure and the difference in pressure makes wind. The wind supplants from low pressure that the more pressure difference results in sever wind the cold and Humid environments are called high pressure regions and Humid regions are called low - pressure. Classification of region in Iran was done by Hasan Ganji which based in Iran is classified to 4 regions: 1) dry and hot regions.2) Humid and hot region 3) Humid and mild 4) cold region [1].

\section{The Features of the Regions, Architecture and Life in Mountainous Areas of Iran}

These areas have average temperature. In the hottest month of the years which is more less than 10 and the average temperature in the coldest month is less than $3 \mathrm{c}$. The characteristics of these areas include long winters, cold and hard that are covered with snow and ice about some months. The cold season starts from early December and lasts decline the cold seasons in these regions. The rainfall level in winter is high and in the summer is low. Regarding the sunshine benefit from the daily fluctuation of temperature, keep the heat and prevent from cold wind in residential environments. It should be mentioned that Kurdistan County that is one of the west county in Iran is located on the high Zagros belt and is one of the high counties in Iran. More than $82 \%$ [2] space of this county such as Bijar is mountainous point despite its dependence to the east, from a small and independent unit with mountains like Naghadeh koub, in west with Nasar. In west south Zagheh kouh and in East with Ghafar khan and khoreh telav. But these mountains aren't very high and relate with their surrounding by some corridors. The Environment Mountains of Bijar in winter are covered with snow and sustain for a long time.

And at last reflection of heat in winters are increased and it helps to settlement of cold weathers aggregation in this area. 
So, Bijar and its environment have particular region conditions in winters. The number of glacial days last $3 / 5$ months and cold reaches to -23 and it has mild summer and in the hot days [3], the temperature reaches to +32 to 40 . The most content of raining is in winter and springs months and the average amount of raining reaches to 437 annually. [3]

\section{General Position of Mountainous Cities}

In modern architecture, the buildings with concrete are metal building, brick coverage and flat and sleepy roofs have been built that don't belong to the areas that are in it and regardless to the regions condition and situation, they use fossil energies for cold and hot that not only cause to irregular consumption and loss of theses energies but also make some problems such as environment pollution, extinction of different ecosystems species, thinning ozone and leads to make the earth hot.

the process of increasing population of cities not with staining the facilities and potentials in the cities existence of natural and human geographical conditions results in physical development of cities. Bijar like other cities meet significant overcrowded and so today this city has encountered so many problems and these problems influence contexture and structure of this city. Overcrowding causes to need to home, increase value of the earth. Development of technology and other economic and social factor which despite of deposit traditional and country materials of these designs, the traditional and compatible designs also are deposited and results in impersonality and un-identity architecture in Bijar which does not have any compatibility with regions and culture situations.

In Bijar nowadays, not main roads and nor secondary roads are not compatible with its region and its squares that had been used for some kinds of application, is used to the cars quartering.

\section{Environmental Accordance of Traditional Textures of Mountainous Cities}

The traditional texture of these areas with concentrated buildings, the compacted and monolith texture show close relationship with each other. In this texture, surfaces of building that suffer from cold are minimized and provide rest and welfare for habitants. These textures have some benefits including easy in movement and availability as well as making regional welfare situation.

Be easy in security in the city and different exit ways in to the texture. [5] Decrease intense effect of cold winds.

In traditional textures, there is an accordance between dimensions and applications and even social attitude and there has not been seen any alienage between apace and operation. In the traditional texture, we have observed correlation which results from connection and adherence of spatial units from two or three dimensioned. Which make the best the regions condition. The traditional textures of this area are introspect which have been made based on the regional meets with, culture and (world view).[5]

\section{Evaluation of Skeletal Elements Identical on the Environment in Building the Traditional Cities in Mountainous Areas}

\subsection{The Residential Buildings in the Mountainous Areas}

In the country homes, often massive wall has been used to build a building which has minimum surface area, the minimum opening and maximum insulation. Besides the plane of this area is compacted and condensed and uses flat roofs to keep snow can be as a heating insulation. Regarding to the severe cold and to receive sun energy for heating, the outer surfaces of the buildings are dark [6]. Based on the cold and mountainous regional situations in Bijar, the buildings are compacted and to prevent loss of the heating energy in it, the outer massive walls that are made of heavy material such as brick. But stone and the windows are located in parasol parts. The houses are adhered and surrounded each other and the roads are narrow to prevent the in the city the material in mountainous areas.

\subsection{Materials in Mountainous Areas}

In the country architectures, due to the use of native material which is the main features of Iranian architecture, the sustainable index of coordinated material with the environment is clear the usage case in rural buildings in Kurdistan like the other regional areas is of available material in that region the body of these buildings are from stone, and coverage of its roof and ceiling is from wood bars and thatch. These materials have good heating resistance and capability to keep the heat inside. the stone and resistant and heavy materials are used to foundation of the building and in some areas, stooling with heavy materials prevents the moisture although the building of these regions are built on the earth, in this region, stone building .make city contexture compatible. The stone which has been found in the area abundant and has used as a bier or shapes in thick walls in the buildings. In this region, the roof of the buildings are flat and covered from wood and thatch beam. The bat walls are abundant in this region. The best is a suitable insulation from heat and cold. Keep the temperature and transit it less. [7]

\section{Incompatibility Modern Texture of the Mountainous Areas Cities with the Environment}

Unlike the traditional textures, the modern textures don't have any concordance with the environment and cannot make 
suitable ecosystem without desirable ventilation and very much energy [5]. The other residential settings haven't been made against the in suitable elements and roof light and the windows not only haven't been made of the society beliefs but also haven't built in accordance with the environment.

Because there has been many attention to build traditional windows, while the big windows are the main reason of cold transit nowadays and unlike the traditional houses which have been opened inside and are kept from in suitable winds damage, these windows are opened to the wide streets and suffer from unsuitable winded and polluted air [5].

In modern textures, the components aren't relate to each other and are made adversely so that there hasn't seen any compatibility among the buildings and spaces.

In last, The materials were used based on their heat capability while nowadays, considering the suitable materials in building or parking have been observed and obey of fashion even in some cases that make the environment, unsuitable is allowable.

The flat roofs with tar and as phaltum coverage and thin walls have improper face even though they have many windows and open areas inside the city that caused to development of improper places in our city. Make wide the streets regardless the environmental features, none existence of proper rhythm in buildings height make our city unfit [5].

\section{Discussion and Conclusion}

The body of these buildings is from stone, and coverage of its roof and ceiling is from wood bars and thatch. These materials have good heating resistance and capability to keep the heat inside the stone and resistant and heavy materials are used to foundation of the moisture. Although the buildings of these regions are built in the earth, in these regions, stone buildings make city contexture compatible. The stone which has been food in this area abundant and has been used as a bier or ship in thick walls in flat and covered from wood and thatch beam. The bat walls are abundant in this region. The best is a suitable insulation from heat and cold, jeep the temperature and transit it less. Incompatibility modern textures of the mountainous areas cities with the environment.

\section{References}

[1] Shakiba manesh, Amir., Mahmoudi, Korush., regulation of environment conditions volt, Tehran pub Tahan haleh, 2011.

[2] Statistical annals of Kurdistan 2007, planning assistant of kordistan governor, 2007.

[3] Koosha, Mohammad Ali, Bijar grous and has an Abad Yasukand. Tehran, sirvan pub, 2006.

[4] Sam .C.M ., Chung, K.P.; Climatic data for building energy design in Hong Kong and mainland China, In proc; of the CIBSE National Conference 1997, London, 1997.

[5] Share rain, Reza, Regional and architecture, Tehran, simaye danesh, 2010.

[6] Alphonso, Mahmoud. Setting the environmental conditions, simaye danesh, 2010.

[7] Ghobadian, Vahid. The regional evaluation of traditional buildings in Iran, Tehran, 2006.

[8] Tahbaz Mansoureh, Jalilian Shahrbanoo. Basis of architecture designation in accordance with strategy for mosque architecture, Tehran, Shahid Beheshti pub, 2009.

[9] Kefa, R. Development of energy - efficient passive solar building design in Nicosia Cyprus; Department of physics; Eastern Mediterranean Uuniversity, Gazimagusa, North Cyprus, Via Mersinlo, Turkey, 2004.

[10] Toy S., Yilmaz S., Yilmaz h.; Determination of bioclimatic comfort in three different land uses in the city of Erzurum, Turkey; Building and Environment, Vol. 42, 2007.

[11] Johansson E, Influence of Urban Geometry on Outdoor Thermal Comfort in a Hot Dry Climate: A Study in Fez, Morocco, Building and Environment 41 (2006) 1326-1338. 